\title{
FRICTIONLESS CONTACT BETWEEN A RIGID INDENTOR AND A TRANSVERSELY ISOTROPIC FUNCTIONALLY GRADED LAYER
}

\author{
R. PATRA \\ Department of Mathematics, Hooghly Engineering and Technology College \\ Vivekananda Road, Hooghly-712103, INDIA \\ E-mail:patra_rajesh@yahoo.co.in \\ S.P. BARIK ${ }^{*}$ \\ Department of Mathematics, Gobardanga Hindu College \\ 24-Parganas (N), Pin-743273, INDIA \\ E-mail:spbarik1@gmail.com \\ P.K. CHAUDHURI \\ Department of Applied Mathematics, University of Calcutta \\ 92, A. P. C. Road, Kolkata-700009, INDIA \\ E-mail: pranay_chaudhuri@yahoo.co.in
}

\begin{abstract}
This article is concerned with the study of frictionless contact between a rigid punch and a transversely isotropic functionally graded layer. The rigid punch is assumed to be axially symmetric and is supposed to be pressing the layer by an applied concentrated load. The layer is resting on a rigid base and is assumed to be sufficiently thick in comparison with the amount of indentation by the rigid punch. The graded layer is modeled as a non-homogeneous medium. The relationship between the applied load $P$ and the contact area is obtained by solving the mathematically formulated problem through using the Hankel transform of different order. Numerical results have been presented to assess the effects of functional grading of the medium and the applied load on the stress distribution in the layer as well as on the relationship between the applied load and the area of contact.
\end{abstract}

Key words: functionally graded material, transversely isotropic medium, Hankel transform, contact problem, Fredholm integral equation.

\section{Introduction}

When a deformable solid is pressed onto another solid, then depending upon the nature and shapes of the solids as well as on the intensity of the applied load, the area of contact between the solids changes and there occurs a significantly different distribution of stress within and outside of the contact area. The determination of the stresses within and outside of the contact area as well as the relationship between the applied load and contact area dimension has been the subject of study in solid mechanics for a long time which started through the initial investigation of Hertz [1] in 1882. A change in the area of contact due to compressive loading on two bodies in contact depends largely on the shapes of the bodies. Contact areas may increase, decrease and sometimes may even remain stationary. Accordingly, contact problems have been classified as advancing (increase of contact area), receding (decrease of contact area) and stationary (contact area remaining the same). A second kind of classification of contact problems, namely, frictional or frictionless contact problems, may also be made based on the consideration of frictional force at the contact

\footnotetext{
* To whom correspondence should be addressed
} 
surface. However, in our present discussion we shall assume that the contact surfaces are smooth and there is no frictional force there. Since contact is the principal method of applying loads to a deformable body, study of contact problems in various kinds of deformable media is important as well as necessary. Owing to their applications in a great variety of structural systems, such as foundations, pavements in roads and runways, automotive disk brake systems and in many other technological applications, considerable progress has been made with the analysis of contact problems in solid mechanics. Among several works done, we may mention a few: Johnson [2]; Gladwell [3]; Hills et al. [4]; Raous et al. [5]; Barik et al. [6, 7, 8]; Chaudhuri and Ray [9]; Comez et al. [10, 11]; Gecit [12]; Fabrikant [13]. Various types of contact problems are discussed in books and journals, e.g. Kit et al. [14]; Ke et al. [15, 16, 17, 18]; Nowacki [19]; Birinci et al. [20]; El-borgi et al. [21, 22]; Patra et al. [23, 24]; Jing et al. [25]; Yan et al. [26]; Adiyaman et al. [27], Jie et al. [28], Giannakopoulos and Suresh [29, 30], Suresh [31].

Most of the solids are supposed to be elastically homogeneous in the sense that elastic coefficients have constant values for a particular solid but vary from one solid to another. Besides, there exists a class of materials in which the elastic coefficients are position dependent in the solid. This class contains lots of materials which exist naturally and also materials artificially manufactured as per need. In solid mechanics, many of the engineering materials, such as composites and a large variety of bonded materials and structural components, are generally modeled as non-homogeneous continua. A functionally graded material (FGM) is a new kind of a non-homogeneous composite in which a gradual change in the volume fraction of constituents from one location to an other in a component. The concept of FGMs was initially proposed in 1984 by a group of scientists in Sendai, Japan (Yamanouchi et al. [32]; Koizumi [33]). Since then, considerable attention has been paid to intensive research on FGMs. Due to the continuously varying material properties in space on the macroscopic scale, FGMs are usually superior to conventional fibrematrix materials in mechanical behavior, especially under thermal loads. It has also been found that properly treated composite materials or functionally graded or non-homogeneous materials are a better choice for their strength and stability in comparison with isotropic and homogeneous materials. This observation demands investigations of various problems in functionally graded media with those characteristics.

The present investigation aims to find the elastostatic solution of an axially symmetric frictionless contact between a transversely isotropic layer and a rigid cylindrical, spherical and conical indentors which are loaded by a concentrated force $P$. Using the operator theory, we derive a general solution that is expressed in terms of the three potentials. These functions satisfy differential equations of the second order and are quasi-harmonic functions. Making use of these fundamental solutions, the punch problem in the aforesaid three cases, is investigated. The solution of the problem has been reduced to the solution of one Fredholm type integral equation of second kind which requires numerical treatment. The numerical results are discussed and presented graphically to show the influence of indentation in the layer on various states of interest.

\section{Formulation of the problem}

We consider a graded layer of transversely isotropic material and of thickness $H$ lying on a rigid base. On the free surface of the layer, a rigid punch of axisymmetric character is placed with its axis of symmetry normal to the free surface of the layer. We also assume that the punch is pressed towards the layer by an applied concentrated force of magnitude $P$. The cylindrical coordinate system $(r, \theta, z)$ with the $z$-axis along the inward drawn normal to the free surface of the layer will be used to specify the position of a point in the layer.

We shall make the following assumptions in our discussion:

(a) the axis of symmetry of the transversely isotropic material is along the $z$-axis,

(b) there is no force of gravity,

(c) linear theory of elasticity holds,

(d) the thickness of the layer is sufficiently large in comparison to the indented depth of the punch. 
We also suppose that for the graded layer, the material is modeled as a nonhomogeneous transversely isotropic material with a gradient oriented along the $z$-direction. The functional grading is assumed to follow the law

$$
A_{i j}(z)=A_{i j}^{0} \cdot \exp (\alpha z), \quad 0<z<H
$$

where $A_{i j}(z)$ are the anisotropic coefficients of the graded medium and $A_{i j}^{0}$ are those for the homogeneous medium and $\alpha$ is a real number.

Because of axisymmetric structure of the indentor, the field variables will be independent of $\theta$ and the displacement vector $(u, 0, w)$ will be function of $r$ and $z$ only. The geometry of the problem is shown in Figs 1a, b, c.

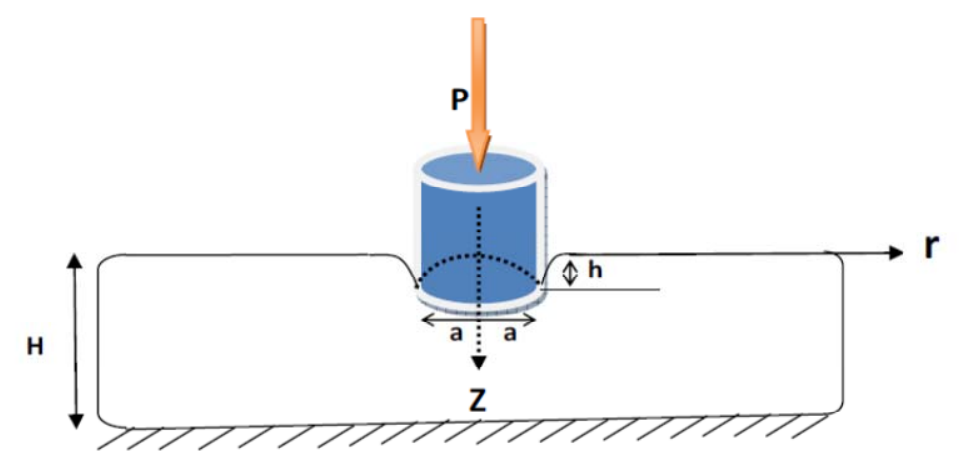

Fig.1a. Geometry of the problem for a flat ended cylindrical punch.

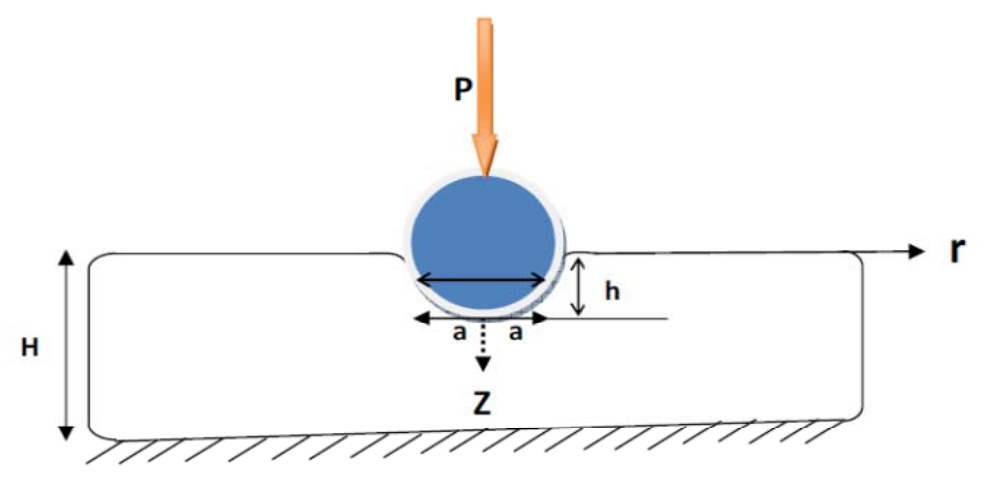

Fig.1b. Geometry of the problem for a spherical punch.

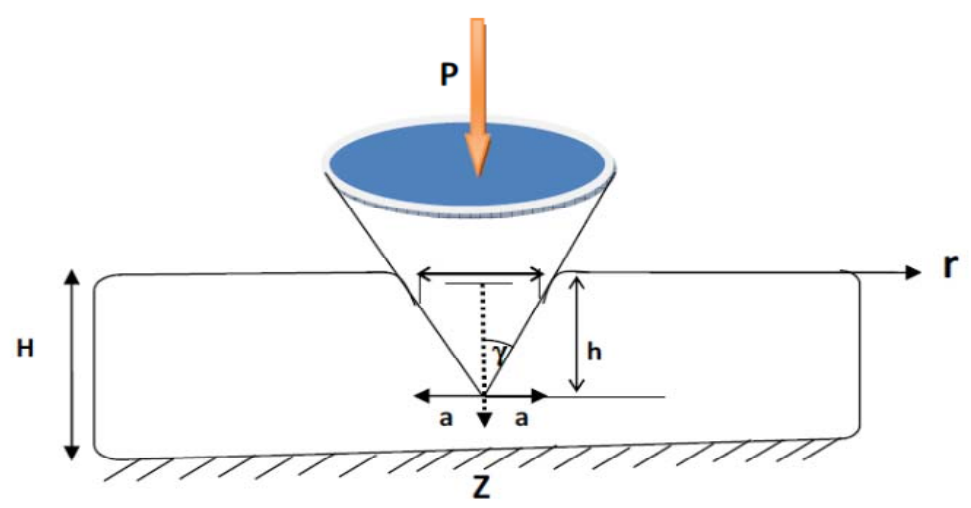

Fig.1c. Geometry of the problem for a conical punch. 
The strain displacement relations, linear stress-strain relations and equations of equilibrium (Lekhnitskii [34]) are respectively given by

$$
\begin{aligned}
& e_{r r}=\frac{\partial u}{\partial r}, \quad e_{\theta \theta}=\frac{u}{r}, \quad e_{z z}=\frac{\partial w}{\partial z}, \quad 2 e_{r z}=\frac{\partial u}{\partial z}+\frac{\partial w}{\partial r}, \\
& {\left[\begin{array}{l}
\sigma_{r r} \\
\sigma_{\theta \theta} \\
\sigma_{z z} \\
\sigma_{z r}
\end{array}\right]=\left[\begin{array}{cccc}
A_{11} & A_{12} & A_{13} & 0 \\
A_{12} & A_{11} & A_{13} & 0 \\
A_{13} & A_{13} & A_{33} & 0 \\
0 & 0 & 0 & A_{44}
\end{array}\right]\left[\begin{array}{c}
e_{r r} \\
e_{\theta \theta} \\
e_{z z} \\
e_{r z}
\end{array}\right],} \\
& \frac{\partial \sigma_{r r}}{\partial r}+\frac{\partial \sigma_{r z}}{\partial z}+\frac{1}{r}\left(\sigma_{r r}-\sigma_{\theta \theta}\right)=0, \\
& \frac{\partial \sigma_{r z}}{\partial r}+\frac{\partial \sigma_{z z}}{\partial z}+\frac{\sigma_{r z}}{r}=0 .
\end{aligned}
$$

Before further proceeding, it will be convenient to adopt non-dimensional variables by rescalling all lengths by the problem's length scale $a$

$$
\begin{aligned}
& r^{\prime}=\frac{r}{a}, \quad z^{\prime}=\frac{z}{a}, \quad w^{\prime}=\frac{w}{a}, \quad u^{\prime}=\frac{u}{a}, \quad H^{\prime}=\frac{H}{a}, \quad h^{\prime}=\frac{h}{a}, \\
& \hat{\sigma}_{i j}=\frac{\sigma_{i j}}{A_{44}^{0}}, \quad(i, j=r, \theta, z), \quad \hat{A}_{1 j}=\frac{A_{1 j}}{A_{44}^{0}}, \quad(j=1,2,3), \quad \hat{A}_{33}=\frac{\mathrm{A}_{33}}{\mathrm{~A}_{44}^{0}} .
\end{aligned}
$$

In the analysis below, for notational convenience, we shall use only non-dimensional variables and politely remove their dashes $(')$ and hats $\left({ }^{\wedge}\right)$.

The equation of equilibrium in terms of nondimensional displacement components $u$ and $w$ may be expressed as follows

$$
\begin{aligned}
& c_{1}\left\{\frac{\partial^{2} u}{\partial r^{2}}+\frac{1}{r} \frac{\partial u}{\partial r}-\frac{u}{r^{2}}\right\}+2 \frac{\partial^{2} w}{\partial r \partial z}+c_{2} \frac{\partial^{2} u}{\partial z^{2}}+\alpha c_{2}\left(\frac{\partial u}{\partial z}+\frac{\partial w}{\partial r}\right)=0, \\
& c_{3} \frac{\partial^{2} w}{\partial z^{2}}+\frac{2}{r} \frac{\partial u}{\partial z}+2 \frac{\partial^{2} u}{\partial r \partial z}+c_{2}\left\{\frac{\partial^{2} w}{\partial r^{2}}+\frac{1}{r} \frac{\partial w}{\partial r}\right\}+\alpha c_{4}\left(\frac{\partial u}{\partial r}+\frac{u}{r}\right)+\alpha c_{3} \frac{\partial w}{\partial z}=0
\end{aligned}
$$

where

$$
c_{1}=\frac{4 A_{11}}{1+2 A_{13}}, \quad c_{2}=\frac{2}{1+2 A_{13}}, \quad c_{3}=\frac{4 A_{33}}{1+2 A_{13}}, \quad c_{4}=\frac{4 A_{13}}{1+2 A_{13}} .
$$

The boundary conditions for the problem are

$$
\begin{aligned}
& w(r, 0)=f(r), \quad 0 \leq r \leq 1, \\
& w(r, H)=0, \quad r \geq 0,
\end{aligned}
$$




$$
\begin{array}{ll}
\sigma_{r z}(r, 0)=0, & r \geq 0, \\
\sigma_{z z}(r, 0)=0, & r>1, \\
\sigma_{r z}(r, H)=0, & r \geq 0 .
\end{array}
$$

In addition to the boundary conditions, the displacement components should satisfy the regularity condition $u, w \rightarrow 0$ as $\sqrt{r^{2}+z^{2}} \rightarrow \infty$. At the surface of contact of the material with the indentor $0 \leq r \leq 1$, the boundary condition will depend upon the shape of the indentor. If $h$ is the indented depth of the solid into the material then

(a) for a cylindrical indentor the condition will be

$$
w(r, 0)=f(r)=h
$$

(b) for a conical indentor having $\gamma$ as the semi-vertical angle, the condition is

$$
w(r, 0)=f(r)=h-(a-r) \cot \gamma .
$$

(c) for a spherical indentor having radius $R$, the condition is

$$
w(r, 0)=f(r)=h-\frac{r^{2}}{2 R}
$$

\section{Method of solution}

To solve the partial differential Eqs (2.7) and (2.8) we shall apply Hankel transforms of order zero and one with respect to the variable $r$. Utilizing the symmetry condition, we shall suppose that the displacement components $u$ and $w$ can be expressed as

$$
\begin{aligned}
& u(r, z)=\int_{0}^{\infty} F_{l}(z, \rho) \rho J_{l}(r \rho) d \rho, \\
& w(r, z)=\int_{0}^{\infty} F_{2}(z, \rho) J_{0}(r \rho) d \rho
\end{aligned}
$$

where $J_{0}$ and $J_{1}$ are Bessel functions of first kind of orders zero and one respectively and the unknown functions $F_{1}(z, \rho), F_{2}(z, \rho)$ are to be determined from the boundary conditions. Substituting from Eqs (3.1) and (3.2) into Eqs (2.7) and (2.8) and inverting the related Hankel transforms we find

$$
\begin{aligned}
& \left\{c_{2} D^{2}+\alpha c_{2} D-c_{1} \rho^{2}\right\} F_{1}-\left\{2 D+\alpha c_{2}\right\} F_{2}=0, \\
& \left\{c_{3} D^{2}+\alpha c_{3} D-c_{2} \rho^{2}\right\} F_{2}+\rho^{2}\left\{2 D+\alpha c_{4}\right\} F_{1}=0
\end{aligned}
$$


where $\quad D=\frac{d}{d z}$.

In deriving Eqs (3.3) and (3.4) the following relationships have been used

$$
\begin{aligned}
& \left\{\frac{\partial^{2}}{\partial r^{2}}+\frac{1}{r} \frac{\partial}{\partial r}-\frac{1}{r^{2}}\right\} J_{l}(r \rho)=-\rho^{2} J_{l}(r \rho), \\
& \left\{\frac{\partial}{\partial r}+\frac{1}{r}\right\} J_{l}(r \rho)=\rho J_{0}(r \rho), \\
& \frac{\partial^{2}}{\partial r^{2}} J_{0}(r \rho)=\frac{\rho}{r} J_{l}(r \rho)-\rho^{2} J_{0}(r \rho) .
\end{aligned}
$$

The solutions of the system of differential Eqs (3.3) and (3.4) are found to be

$$
\begin{aligned}
& F_{1}(z, \rho)=\sum_{k=1}^{4} A_{k}(\rho) e^{m_{k} z} \\
& F_{2}(z, \rho)=\sum_{k=1}^{4} a_{k}(\rho) A_{k}(\rho) e^{m_{k} z}
\end{aligned}
$$

where $A_{k}(\rho)(k=1, \ldots, 4)$ are arbitrary functions of the transformed parameter $\rho$, which are to be determined from the boundary conditions $(2.10)$ - (2.14) and $m_{k}(k=1, \ldots . .4)$ are the roots of the following characteristic equation

$$
m^{4}+2 \alpha m^{3}+\left(\alpha^{2}-2 \delta \rho^{2}\right) m^{2}-2 \alpha \delta \rho^{2} m+\beta_{2} \alpha^{2} \rho^{2}+\beta_{0}^{2} \rho^{4}=0
$$

with

$$
\begin{aligned}
& \frac{c_{1} c_{3}+c_{2}^{2}-4}{c_{3}}=\frac{c_{1} c_{3}+c_{2}^{2}-2 c_{2}-2 c_{4}}{c_{3}}=\delta,(\text { say }), \\
& \beta_{0}^{2}=\frac{\mathrm{c}_{1}}{\mathrm{c}_{3}}, \quad \beta_{2}=\frac{c_{4}}{c_{3}},
\end{aligned}
$$

and the coefficients $a_{k}(k=1, \ldots ., 4)$ are given by

$$
a_{k}(\rho)=-\rho^{2} \frac{2 m_{k}+\alpha c_{4}}{c_{3}\left(m_{k}^{2}+\alpha m_{k}\right)-c_{2} \rho^{2}}, \quad(k=1, \ldots ., 4) .
$$

It follows from Eq.(3.8) that 


$$
\begin{aligned}
& m_{1}=\bar{m}_{3}=-\frac{\alpha}{2}+\sqrt{\rho^{2}+\frac{\alpha^{2}}{4}+i \rho \sqrt{\beta_{2} \alpha^{2}-\beta_{l}^{2} \rho^{2}}}, \\
& m_{2}=\bar{m}_{4}=-\frac{\alpha}{2}-\sqrt{\rho^{2}+\frac{\alpha^{2}}{4}+i \rho \sqrt{\beta_{2} \alpha^{2}-\beta_{l}^{2} \rho^{2}}},
\end{aligned}
$$

with

$$
\beta_{1}^{2}=\delta^{2}-\beta_{0}^{2}
$$

where $\bar{m}_{3}, \bar{m}_{4}$ denote the respective complex conjugates of $m_{1}, m_{2}$.

Using Eqs (3.6), (3.7), (3.1) and (3.2) in the boundary conditions (2.10) - (2.14) we obtain

$$
\begin{aligned}
& \sum_{k=1}^{4}\left[m_{k}(\rho)-a_{k}(\rho)\right] A_{k}(\rho)=0, \quad r \geq 0, \\
& \sum_{k=1}^{4}\left[m_{k}(\rho)-a_{k}(\rho)\right] A_{k}(\rho) e^{m_{k}(\rho) H}=0, \quad r \geq 0, \\
& \sum_{k=1}^{4} a_{k}(\rho) A_{k}(\rho) e^{m_{k}(\rho) H}=0, \quad r \geq 0, \\
& \int_{0}^{\infty} \sum_{k=1}^{4} a_{k}(\rho) A_{k}(\rho) J_{0}(r \rho) d \rho=f(r), \quad 0 \leq r \leq 1, \\
& \int_{0}^{\infty} \sum_{k=1}^{4}\left[A_{13}^{\prime}(\rho) \rho^{2}+A_{33}^{\prime}(\rho) a_{k}(\rho)\right] A_{k}(\rho) J_{0}(r \rho) d \rho, \quad r>1 .
\end{aligned}
$$

Equatios (3.14) - (3.16) yield

$$
A_{k}(\rho)=g_{k}(\rho) A_{4}(\rho), \quad(\mathrm{k}=1,2,3)
$$

where

$$
\begin{gathered}
g_{1}(\rho)=\frac{1}{|A|}\left|\begin{array}{ccc}
-b_{4} & b_{2} & b_{3} \\
-b_{4} e^{m_{4} H} & b_{2} e^{m_{2} H} & b_{3} e^{m_{3} H} \\
-a_{4} e^{m_{4} H} & a_{2} e^{m_{2} H} & a_{3} e^{m_{3} H}
\end{array}\right|, \\
g_{2}(\rho)=\frac{1}{|A|}\left|\begin{array}{ccc}
b_{1} & -b_{4} & b_{3} \\
b_{1} e^{m_{1} H} & -b_{4} e^{m_{4} H} & b_{3} e^{m_{3} H} \\
a_{1} e^{m_{1} H} & -a_{4} e^{m_{4} H} & a_{3} e^{m_{3} H}
\end{array}\right|,
\end{gathered}
$$




$$
\begin{aligned}
& g_{3}(\rho)=\frac{1}{|A|}\left|\begin{array}{ccc}
b_{1} & b_{2} & -b_{4} \\
b_{1} e^{m_{1} H} & b_{2} e^{m_{2} H} & -b_{4} e^{m_{4} H} \\
a_{1} e^{m_{1} H} & a_{2} e^{m_{2} H} & -a_{4} e^{m_{4} H}
\end{array}\right|, \\
& |A|=\left|\begin{array}{ccc}
b_{1} & b_{2} & b_{3} \\
b_{1} e^{m_{1} H} & b_{2} e^{m_{2} H} & b_{3} e^{m_{3} H} \\
a_{1} e^{m_{1} H} & a_{2} e^{m_{2} H} & a_{3} e^{m_{3} H}
\end{array}\right|,
\end{aligned}
$$

and

$$
b_{k}=m_{k}(\rho)-a_{k}(\rho), \quad(k=1, \ldots ., 4) \text {. }
$$

From Eqs (3.18) and (3.17) we get, respectively

$$
\begin{aligned}
& \int_{0}^{\infty} D(\rho) A_{4}(\rho) J_{0}(r \rho) d \rho=0, \quad r>1, \\
& \int_{0}^{\infty} D^{*}(\rho) A_{4}(\rho) J_{0}(r \rho) d \rho=f(r), \quad 0 \leq r \leq 1,
\end{aligned}
$$

with

$$
\begin{aligned}
& D(\rho)=\sum_{k=1}^{3} l_{k}(\rho) g_{k}(\rho)+l_{4}(\rho), \\
& l_{k}(\rho)=A_{13}^{\prime} \rho^{2}+A_{33}^{\prime} a_{k}(\rho), \\
& D^{*}(\rho)=\sum_{k=1}^{3} a_{k}(\rho) g_{k}(\rho)+a_{4}(\rho) .
\end{aligned}
$$

If we write $\quad B(\rho)=D(\rho) A_{4}(\rho)$,

Equations (3.21) and (3.22) respectively become

$$
\begin{aligned}
& \int_{0}^{\infty} B(\rho) J_{0}(r \rho) d \rho=0, \quad r>1, \\
& \int_{0}^{\infty} G(\rho) B(\rho) J_{0}(r \rho) d \rho=f(r), \quad 0 \leq r \leq 1
\end{aligned}
$$

where $\quad G(\rho)=\frac{D^{*}(\rho)}{D(\rho)}$.

Introducing a function $\phi(t)$ such that 


$$
\frac{B(\rho)}{\rho}=\sqrt{\frac{2}{\pi}} \int_{0}^{1} \phi(t) \cos (\rho t) d t
$$

we find that Eq.(3.27) is automatically satisfied and we get

$$
A_{4}(\rho)=\frac{\rho}{D(\rho)} \sqrt{\frac{2}{\pi}} \int_{0}^{1} \phi(t) \cos (\rho t) d t .
$$

Now Eq.(3.28) can be written as

$$
\int_{0}^{r} \frac{d t}{\sqrt{r^{2}-t^{2}}}\left[\phi(t)+\int_{0}^{l} \phi(x) L(x, t) d x\right]=f(r), \quad 0 \leq r \leq 1,
$$

which is an Abel type integral equation. After some calculations we get the integral equation in $\phi$ as

$$
\phi(t)+\int_{0}^{l} \phi(x) L(x, t) d x=\frac{2}{\pi} g(t), \quad 0 \leq t \leq 1 .
$$

This equation determines the function $\phi$. Here

$$
\begin{aligned}
& g(t)=\frac{d}{d t} \int_{0}^{t} \frac{r f(r)}{\sqrt{t^{2}-r^{2}}} d r=h, \quad \text { for cylindrical indentor, } \\
& =h+\left(\frac{\pi}{2} t-1\right) \cot \gamma, \quad \text { for conical indentor, } \\
& =h-\frac{t^{2}}{R}, \quad \text { for spherical indentor, } \\
& L(x, t)=\frac{2}{\pi} \int_{0}^{\infty} \Omega(\rho) \cos (\rho x) \cos (\rho t) d \rho, \\
& \Omega(\rho)=\sqrt{\frac{2}{\pi}} \rho G(\rho) .
\end{aligned}
$$

Now the equilibrium condition demand

$$
P^{*}+2 \pi \int_{0}^{a} r \sigma_{z z}(r, 0) d r=0
$$

where

$$
P^{*}=\frac{P}{A_{44}^{0}}, \quad a^{*}=\frac{a}{h},
$$




$$
\begin{aligned}
& \sigma_{z z}(r, 0)=\int_{0}^{\infty} M(\omega) J_{0}(\omega r) d \omega, \\
& M(\omega)=\sqrt{\frac{2}{\pi}} \int_{0}^{1} \phi(t) \cos (\omega t) d \omega t, \quad \omega=\rho h .
\end{aligned}
$$

Equation (3.37) is the relationship between the non-dimensional applied load $P^{*}$ and $a^{*}$, the nondimensional radius of the contact area.

\section{Solution of the integral equations}

The interval $[0,1]$ is sub-divided into $n$ equal parts, each of length $h_{1}$ by the points, $0=x_{0}, x_{1}=x_{0}+h_{1}, x_{2}=x_{0}+2 h_{1}, \ldots \ldots \ldots . ., x_{n}=x_{0}+n h_{1}=1 \quad$ where $n h_{1}=1, \quad$ such $\quad$ that $x_{j}=x_{0}+j h_{1}, j=0,1, \ldots \ldots \ldots, n$. Then by Simpson's $1 / 3$ formula we get

$$
\begin{aligned}
& \int_{0}^{l} \phi(x) L(x, t) d x= \\
& =\frac{h_{1}}{3} \sum_{j=1}^{\frac{n}{2}}\left[\phi\left(x_{2 j-2}\right) L\left(x_{2 j-2}, t_{i}\right)+4 \phi\left(x_{2 j-1}\right) L\left(x_{2 j-1}, t_{i}\right)+\phi\left(x_{2 j}\right) L\left(x_{2 j}, t_{i}\right)\right]
\end{aligned}
$$

where $t_{i}=x_{i}, i=0,1, \ldots \ldots \ldots, n$.

Now, the integral Eq.(3.33) can be written as

$$
\begin{aligned}
& \phi\left(t_{i}\right)+\frac{h_{1}}{3} \sum_{j=1}^{\frac{n}{2}}\left[\phi\left(x_{2 j-2}\right) L\left(x_{2 j-2}, t_{i}\right)+4 \phi\left(x_{2 j-1}\right) L\left(x_{2 j-1}, t_{i}\right)+\phi\left(x_{2 j}\right) L\left(x_{2 j}, t_{i}\right)\right]= \\
& =\frac{2}{\pi} g\left(t_{i}\right), \quad i=0,1, \ldots \ldots, n
\end{aligned}
$$

which is a set of $n$ linear equations in $\mathrm{n}$ unknowns $\phi\left(t_{0}\right), \phi\left(t_{1}\right)$, $\phi\left(t_{n}\right)$. method.

This linear algebraic system of equations are solved numerically by utilizing Gaussian elimination

\section{Numerical results and discussion}

The present study aims at investigating a frictionless contact problem in a finite transversely isotropic functionally graded layer. The main objective of the present discussion is to study the effects of material gradation and indentation on the load-contact area relationship as well as on normal stress distribution.

In our present discussion we have considered the transversely isotropic materials as cobalt, magnesium and titanium to illustrate theoretical results. The numerical values of the elastic coefficients for the materials are listed in Tab.1 (Freund and Suresh [35]). 
Table 1. Basic data for three transversely isotropic materials.

\begin{tabular}{|c|c|c|c|c|}
\hline Quantity & Unit & Cobalt & Titanium & Magnesium \\
\hline$A_{11}$ & $\mathrm{GPa}$ & 307.0 & 162.4 & 59.7 \\
$A_{33}$ & $\mathrm{GPa}$ & 358.1 & 180.7 & 61.7 \\
$A_{44}$ & $\mathrm{GPa}$ & 78.3 & 46.7 & 16.4 \\
$A_{12}$ & $\mathrm{GPa}$ & 165.0 & 92.0 & 26.2 \\
$A_{13}$ & $\mathrm{GPa}$ & 103.0 & 69.0 & 21.7 \\
\hline
\end{tabular}

Using the above numerical data we shall evaluate numerically the integral Eq.(3.33). Our numerical study will cover three different types of a rigid indentor, namely, cylindrical shaped, spherical shaped and conical shaped.

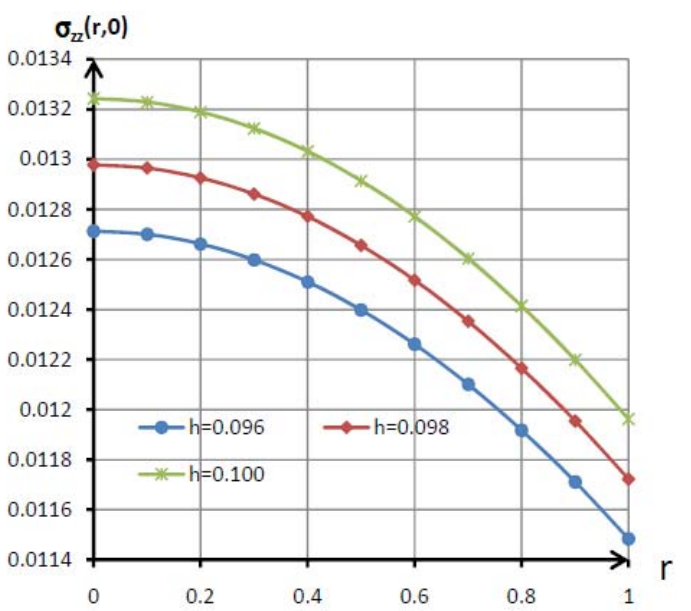

(a)

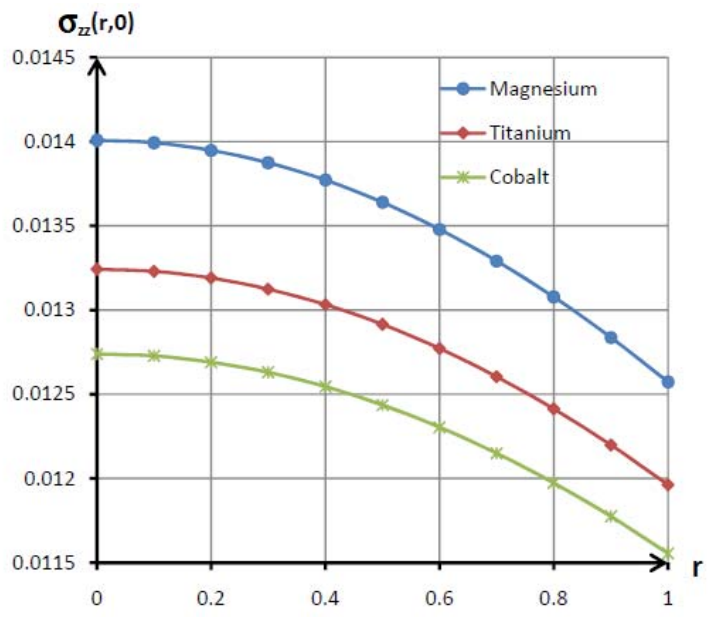

(b)

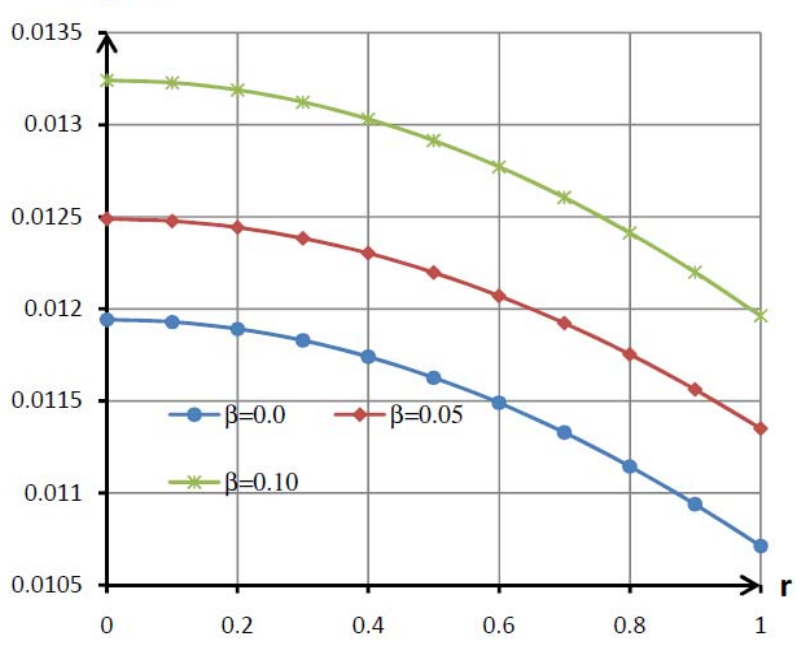

(c)

Fig.2a. The effect of indentation $h$ on $\sigma_{z z}(r, 0)$ for the flat -ended cylindrical punch (Titanium) $(\alpha=0.1)$. (b) Variation of $\sigma_{z z}(r, 0)$ for various materials with fixed indentation of the flat-ended cylindrical punch $(h=0.1, \alpha=0.1)$. (c) The effect of the graded parameter $\alpha$ on $\sigma_{z z}(r, 0)$ for the flat-ended cylindrical punch with $h=0.1$ (Titanium). 
We shall display our numerical results in Figs 2, 3 and 4 corresponding to cylindrical, spherical and conical shaped punches. Figure 2a shows the effect of indentation $h$ on $\sigma_{z z}(r, 0)$ for flat-ended cylindrical punch for various values of $h$ in a transversely isotropic functionally graded medium like titanium. As expected, more indentation will require more normal stress. Figure $2 b$ shows variation of $\sigma_{z z}(r, 0)$ for three different materials with fixed indentation $h$ of the flat-ended cylindrical punch. It is evident from Fig. $2 \mathrm{~b}$ that increase in rigidity will generate less normal stress. Figure $2 \mathrm{c}$ illustrates the effect of graded parameter $\alpha$ on $\sigma_{z z}(r, 0)$. It shows that normal stress $\sigma_{z z}(r, 0)$ increases as the graded parameter $\alpha$ increases.

There are not much significant changes in the behavior of the stresses in the case of the spherical indentor from the corresponding results of the cylindrical punch as shown in Figs.3. But here we see that $\sigma_{z z}(r, 0) \rightarrow 0$ as $r \rightarrow 1$.

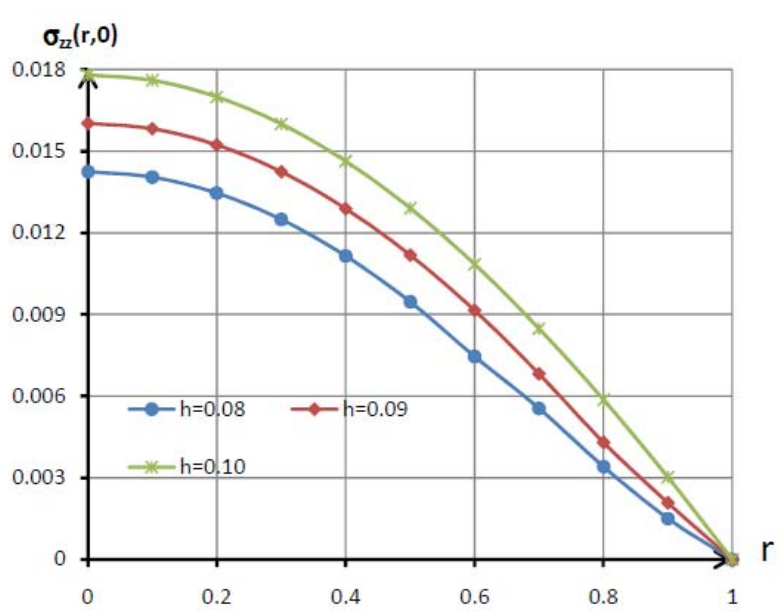

(a)

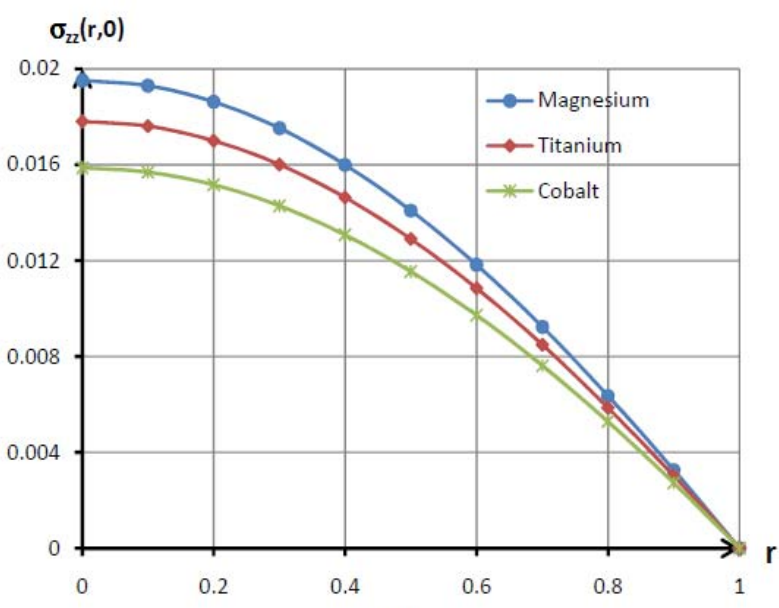

(b)

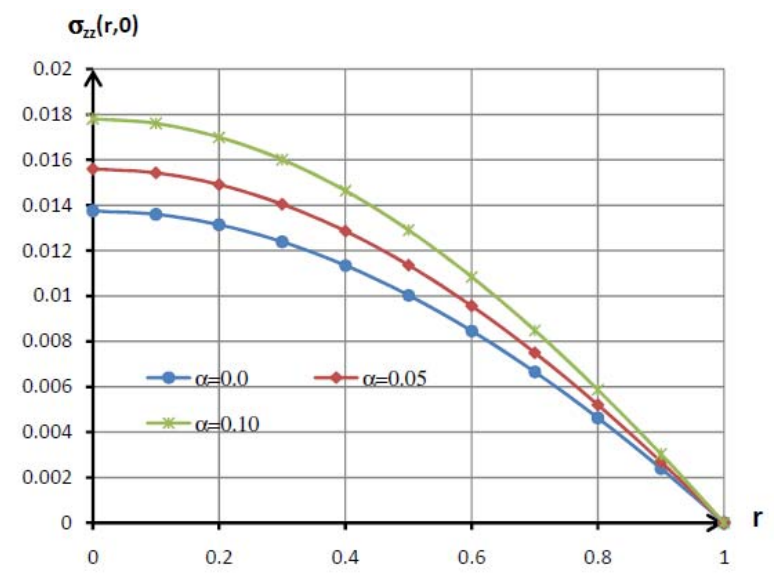

(c)

Fig.3a. The effect of indentation $h$ on $\sigma_{z z}(r, 0)$ for the spherical punch (Titanium) $(\alpha=0.1, R=10)$. (b) Variation of $\sigma_{z z}(r, 0)$ for various materials with fixed indentation of the spherical punch $(h=0.1, \alpha$ $=0.1, R=10)$. (c) The effect of the graded parameter $\alpha$ on $\sigma_{z z}(r, 0)$ for spherical punch with $h=$ $0.1, R=10$ (Titanium).

In the case of the conical punch, some kind of dissimilarities from the above two punches are observed. Firstly, in this case the stresses act oppositely and have decreasing numerical values with 
increasing $h$. This is shown in Fig.4a. Figure $4 \mathrm{~b}$ shows a comparison between results of three materials for a fixed $h$. The results are similar to those in Fig.2b. Figure 4c shows the same effects as those of Figs $2 \mathrm{c}$ and $3 \mathrm{c}$ and e., the numerical values of $\sigma_{z z}(r, 0)$ increase as $\alpha$ increases. Variations of normal stress with $r$ have been also studied with different shapes of the conical punch by varying the semi-vertical angle. Results are shown in Fig.4d.

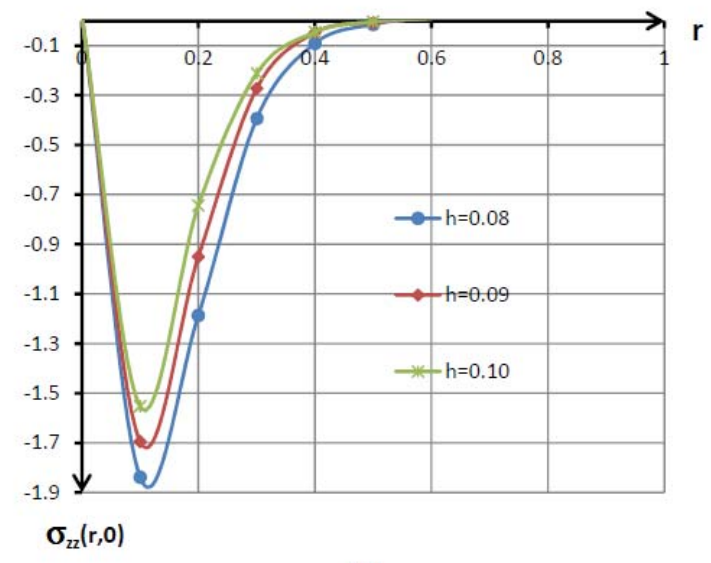

(a)

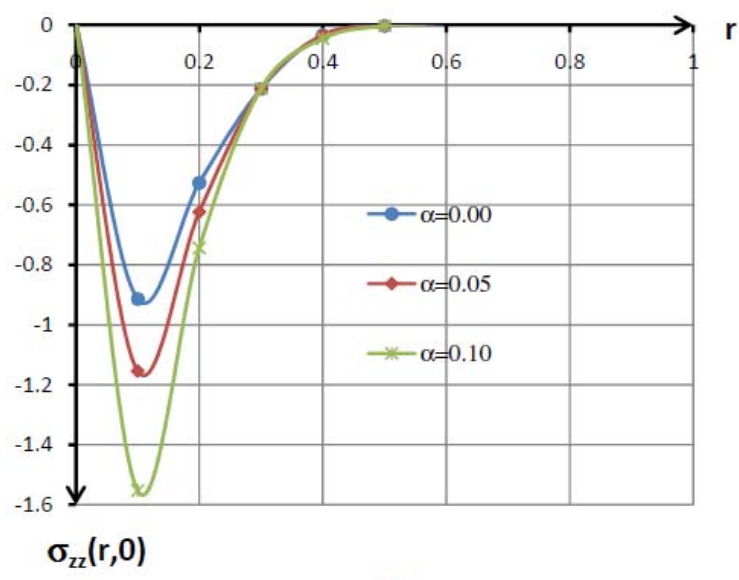

(c)

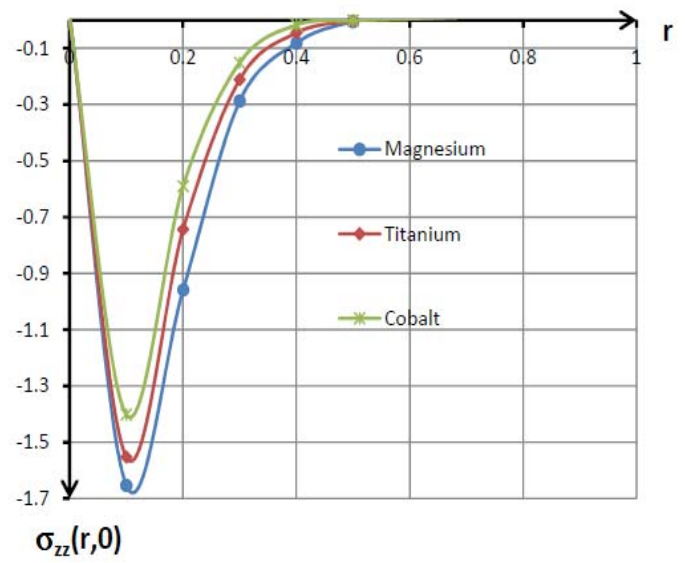

(b)

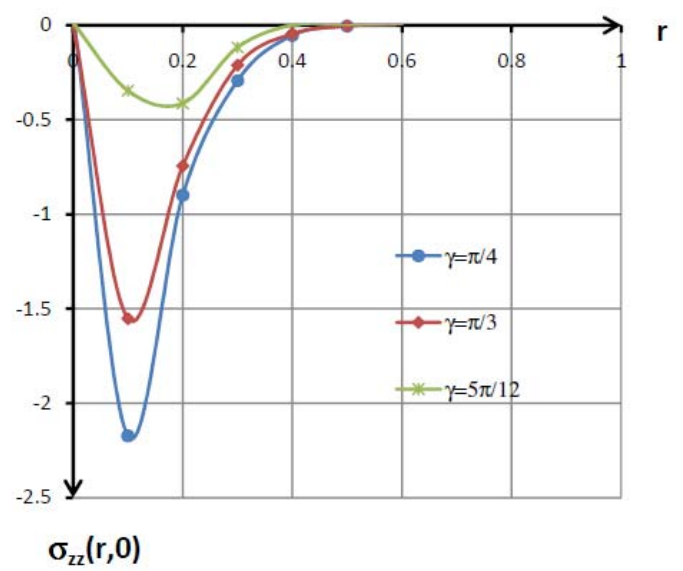

(d)

Fig.4a. The effect of indentation $h$ on $\sigma_{z z}(r, 0)$ for the conical punch (Titanium) $(\alpha=0.1, \gamma=\pi / 3)$. (b) Variation of $\sigma_{z z}(r, 0)$ for various materials with fixed indentation of the conical punch $(h=0.1, \alpha=0.1, \gamma=\pi / 3)$. (c) The effect of graded parameter $\alpha$ on $\sigma_{z z}(r, 0)$ for the conical punch with $h=0.1, \gamma=\pi / 3$ (Titanium). (d) Variation of $\sigma_{z z}(r, 0)$ for different $\gamma$ of the conical punch with $h=0.1, \alpha=0.1$ (Titanium).

Figures 5 shows the variation of the applied load $P^{*}$ with contact radius $a^{*}$ for all kinds of considered punches. Figure 5a shows that to produce same amount of indentation, a cylinder with greater radius will require a greater magnitude of the applied load. For materials like titanium, Figs 5a, c, e show that the effects of the graded parameter $\alpha$ on the applied load-contact area relationship for three different types of punches. It is clear from Figs 5a, c that as rigidity increases, a greater magnitude of the applied load will be required to produce same area of contact for cylindrical and spherical punches, while for the conical punch, as shown in Fig.5e, the result is just the opposite i.e., with increased rigidity, as load increases, contact area diminishes. The effects of anisotropy on the applied load are very much clear in Figs $5 \mathrm{~b}, \mathrm{~d}, \mathrm{f}$ for flat-ended cylindrical, spherical and conical punches respectively. 


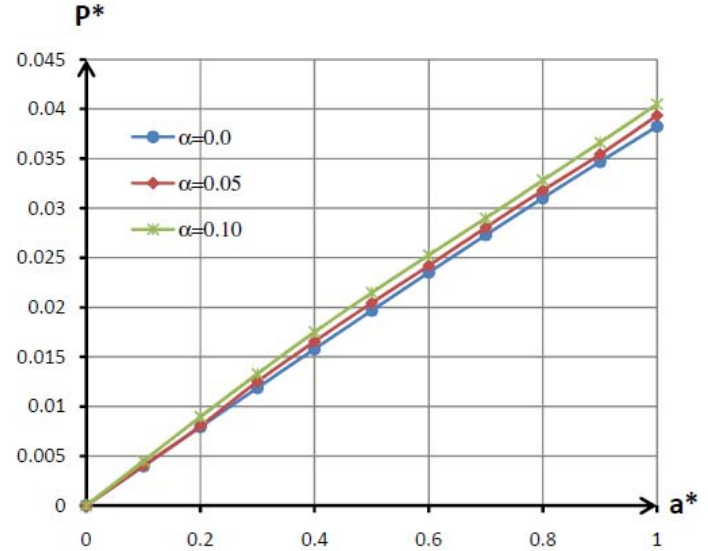

(a)

$$
\text { p* }
$$

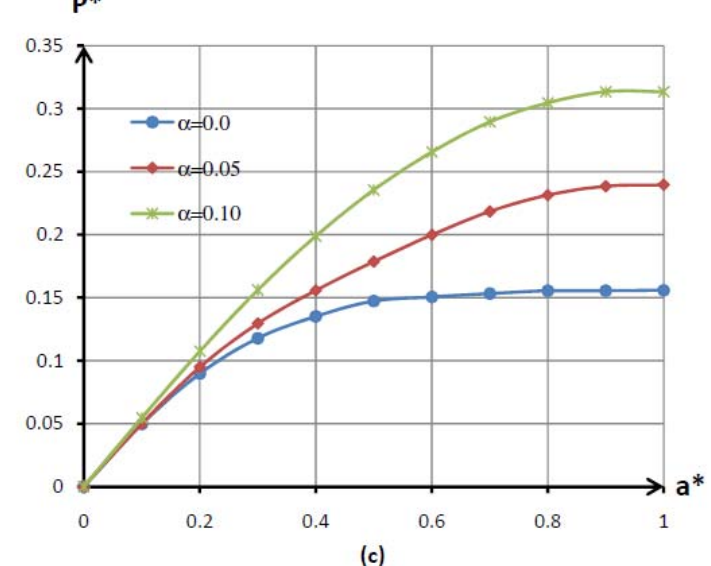

P*

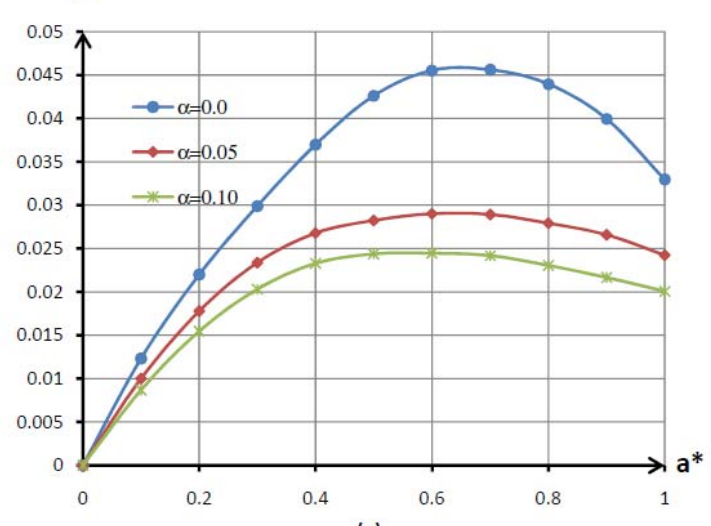

(e)

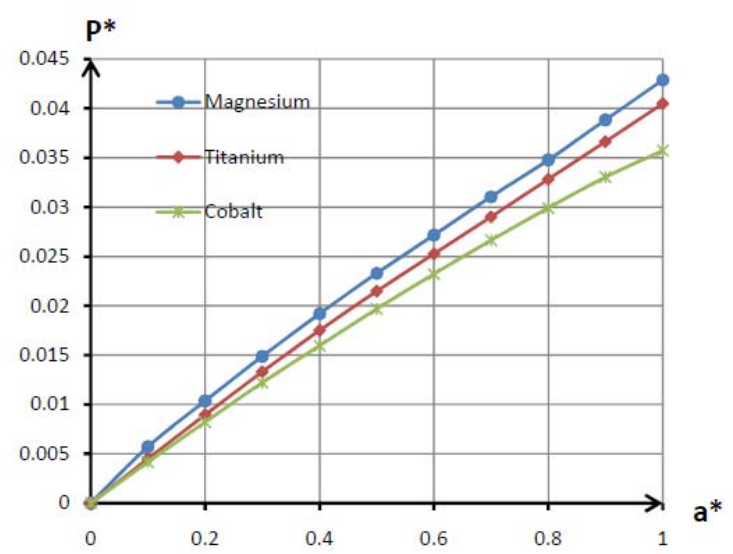

(b)

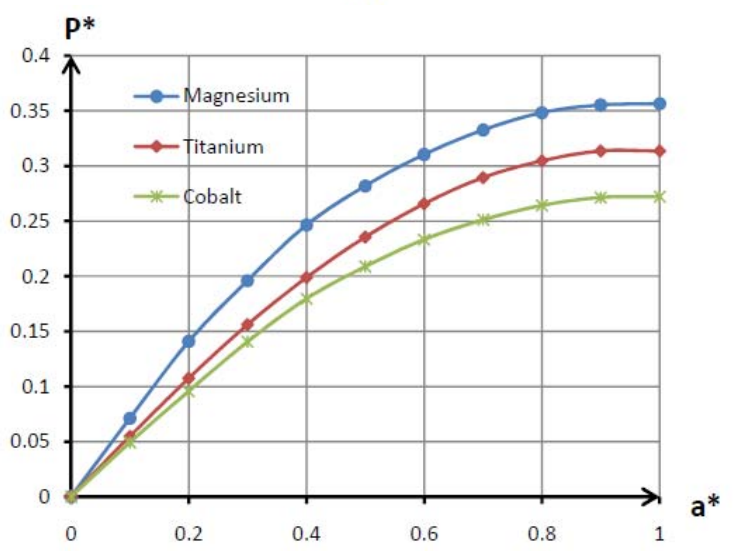

(d)

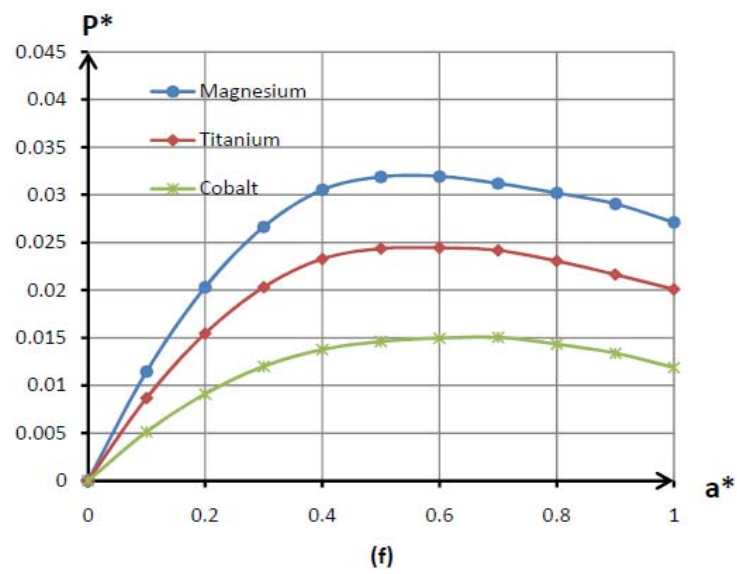

(f)

Fig.5a. The effect of the graded parameter $\alpha$ on total force $P^{*}$ with contact radius with fixed indentation $(h=$ $0.1)$ for the flat-ended cylindrical punch (Titanium). (b) Variation of total force $P^{*}$ with contact radius $(h=0.1, \alpha=0.1)$ for the flat-ended cylindrical punch. (c) The effect of the graded parameter $\alpha$ on total force $P^{*}$ with contact radius with fixed indentation $(h=0.1, R=10)$ for the spherical punch (Titanium). (d) Variation of total force $P^{*}$ with contact radius for various materials $(h=0.1, \alpha=0.1$, $R=10$ ) for the spherical punch. (e) The effect of the graded parameter $\alpha$ on total force $P^{*}$ with contact radius with fixed indentation $(h=0.1, \gamma=\pi / 3)$ for the conical punch (Titanium). (f) Variation of total force $P^{*}$ with contact radius for various materials $(h=0.1, \alpha=0.1, \gamma=\pi / 3)$ for conical punch. 


\section{Conclusion}

In the present study of frictionless contact between a transversely isotropic functionally graded layer and a rigid indentor pushed normally towards the layer, the gradation of the layer, the applied load on the indentor and the shapes of the indentor appear to produce significant effects on the normal stress distribution at the layer surface and on the load-contact area relationship. As, neither the expression of the surface normal stress, nor the load- contact area relationship is in its simple algebraic form, the effects are studied through numerical evaluations of the associated integrals based on the various parameter values of the medium. The important observations from the study are

(a) The normal stress distribution $\sigma_{z z}(r, 0)$ depends on the amount of indentation, the shape of the indentor as well as on the rigidity of the layer material

(b) The load-contact area relationship also depends on the shape of the indentor, the anisotropic character as well as on the functional gradation of the layer material.

\section{Nomenclature}

$$
\begin{aligned}
A & - \text { non homogeneity parameter } \\
A_{i j} & - \text { anisotropic coefficients for the graded elastic medium } \\
A_{i j}^{0} & - \text { anisotropic coefficients for the homogeneous elastic medium } \\
e_{r r}, e_{r z}, e_{\theta \theta}, e_{z z} & - \text { strain components in polar co-ordinate system } \\
H & - \text { thickness of the layer } \\
J_{n}(.) & - \text { bessel functions of first kind with order } n \\
P & - \text { applied concentrated load } \\
R & - \text { radius of spherical indentor } \\
r, \theta, z & - \text { cylindrical coordinates } \\
u, w & - \text { displacement components } \\
\gamma & - \text { semi vertical angle of the conical indentor } \\
\sigma_{r r}, \sigma_{r z}, \sigma_{\theta \theta}, \sigma_{z z} & - \text { stress components in polar co-ordinate system }
\end{aligned}
$$

\section{References}

[1] Hertz H. (1882): On the contact of rigid elastic solids. - J. Reine and Angewandte Mathematik, vol.92, pp.156-171.

[2] Johnson K.L. (1985): Contact Mechanics. - Cambridge: Cambridge University Press.

[3] Gladwell M.L. (1980): Contact Problems in the Classical Theory of Elasticity. - Sijthoff and Noordhoff, The Netherlands.

[4] Hills D.A. and Nowell D. (1993): Sackfield A. Mechanics of Elastic Contacts. - Butterworth Heinemann.

[5] Raous M., Jean M. and Moreau J.J. (1995): Contact Mechanics. - New York: Plenum Press.

[6] Barik S.P., Kanoria M. and Chaudhuri P.K. (2006): Contact problem for an anisotropic elastic layer lying on an anisotropic elastic foundation under gravity. - J. Ind. Acad. Math., vol.28, pp.205-223.

[7] Barik S.P., Kanoria M. and Chaudhuri P.K. (2008): Effect of nonhogeniety on the contact of an isotropic half-space and a rigid base with an axially symmetric reces. - J. Mech. Materials and Struc., vol.3, pp.1-18.

[8] Barik S.P., Kanoria M. and Chaudhuri P.K. (2009): Frictionless contact of a functionally graded half-space and a rigid base with an axially symmetric recess. - J. Mech., vol.25, pp.9-18.

[9] Chaudhuri P.K. and Ray S. (2003): Receding axisymmetric contact between a transversely isotropic layer and a transversely isotropic half-space. - Bull. Cal. Math. Soc., vol.95, pp.151-164. 
[10] Comez I. (2013): Contact problem of a functionally graded layer resting on a Winkler foundation. - Act. Mech., vol.224, pp.2833-2843.

[11] Comez I., El-Borgi S., Kahya V. and Erdöl R. (2016): Receding contact problem for two-layer functionally graded media indented by a rigid punch. - Acta. Mech., vol. 227, pp.2493-2504.

[12] Gecit M.R. (1981): Axisymmetric contact problem for an elastic layer and an elastic foundation. - Int. J. Engg. Sci., vol.19, pp.747-755.

[13] Fabrikant V.I. (2006): Elementary solution of contact problems for a transversely isotropic layer bonded to a rigid foundation. - Z. Angew. Math.Phys., vol.57, pp.464-490.

[14] Kit G.S. and Monastyrsky B.E. (2001): A contact problem for a half-space and a rigid base with an axially symmetric recess. - J. Mathematical Sci., vol.107, pp.3545-3549.

[15] Ke L.L., Yang J., Kitipornchai S. and Wang Y.S. (2008): Frictionless contact analysis of a functionally graded piezoelectric layered half-plane. - Smart Mater. Struc., vol.17, pp.025003.

[16] Ke L.L and Wang Y.S. (2007): Two dimensional sliding frictional contact of a functionally graded materials. European J. Mech. A/Solids., vol.26, pp.171-188.

[17] Ke L.L., Yang J., Kitipornchai S. and Wang Y.S. (2008): Electro-mechanical frictionless contact behavior of a functionally graded piezoelectric layered half-plane under a rigid punch. - Int. J. Solids Struc., vol.4, pp.33133333.

[18] Ke L.L., Yang J., Kitipornchai S. and Wang Y.S. (2010): Sliding frictional contact analysis of functionally graded piezoelectric layered half-plane. - Acta Mech., vol.209, pp.249-268.

[19] Nowacki W. (1973): Theory of Elasticity. - Warsaw: PWN.

[20] Birinci A. and Erdol R. (1999): Frictionless contact between a rigid stamp and an elastic layered composite resting on simple supports. - Math. and Comp. Appl., vol.4, pp.261-272.

[21] El-Borgi S., Abdelmoula R. and Keer L. (2006): A receding contact plane problem between a functionally graded layer and a homogeneous substrate. - Int. J. Solids and Struc., vol.43, pp.658-674.

[22] El-Borgi S., Usman S. and Guler M.A. (2014): A frictional receding contact plane problem between a functionally graded layer and a homogeneous substrate. - Int. J. Solids Struc., vol.51, pp.4462-4476.

[23] Patra R., Barik S.P., Kundu M. and Chauaudhuri P.K. (2014): Plane Elastostatic Solution in an Infinite Functionally Graded Layer Weakened by a Crack Lying in the Middle of the Layer. - Int. J. Computational Math., vol.2014, Article ID 358617.

[24] Patra R., Barik S.P. and Chaudhuri P.K. (2016): Frictionless contact of a rigid punch indenting a transversely isotropic elastic layer. - Int. J. Adv. Appl. Math. and Mech., vol.3, pp.100-111.

[25] Jing L., Ke L.L., Wang Y.S., Yang J. and Alam F. (2012): Thermoelastic frictional contact of functionally graded materials with arbitrarily varying properties. - Int. J. Mech. Sci., vol.63, pp.86-98.

[26] Yan J. and Li X. (2015): Double receding contact plane problem between a functionally graded layer and an elastic layer. - European J. Mech. A/Solids., vol.53, pp.143-150.

[27] Adiyaman G., Birinci A. and Öner E. (2016): A receding contact problem between a functionally graded layer and two homogeneous quarter planes. -Acta. Mech., vol.227, pp.1753-1766.

[28] Jie S. and Liao-Liang Yue-Sheng K.W. (2016): Axisymmetric frictionless contact of a functionally graded piezoelectric layered half-space under a conducting punch. - Int. J. Solids Struc., vol.90, pp.45-59.

[29] Giannakopoulos A.E. and Suresh S. (1997): Indentation of solids with gradients in elastic properties: part I. Point force solution. - Int. J. Solids Struc., vol.34, pp.2357-2392.

[30] Giannakopoulos A.E. and Suresh S. (1997): Indentation of solids with gradients in elastic properties: part II. Axisymmetric indentors. - Int. J. Solids Struc., vol.34, pp.2393-2428. 
[31] Suresh S. (2001): Graded materials for resistance to contact deformation and damage. - Science, vol.292, pp.2447-2451.

[32] Yamanouchi M., Koizumi M. and Shiota (Eds) I. (1990): Proceedings of the 1st international symposium on functionally gradient materials. -Functionally gradient materials forum and the society of non-traditional technology. - Japan.

[33] Koizumi M. (1993): Concept of FGM. - Ceramic Trans., vol.34, pp.3-10.

[34] Lekhnistkii S.G. (1981): Theory of Elasticity of an Anisotropic Body. - Moscow: Mir Publishers.

[35] Freund L.B. and Suresh S. (2003): Thin Film Materials. - CUP.

[36] Gradshteyn I.S. and Ryzhik I.M. (1963): Table of Integrals, Series and Products. - Academic Press.

Received: July 16, 2018

Revised: June 6, 2018 